\title{
Fistula-Related Pericarditis as a Complication of Gastric Bypass Surgery
}

\section{Avinash Jayaswal $^{1 *}$ and Mohamed El Koulali ${ }^{2}$}

${ }^{1}$ Department of Emergency Medicine, Erasme Hospital - ULB, Brussels, Belgium

${ }^{2}$ Department of Gastroenterology, Erasme Hospital - ULB, Brussels, Belgium

*Corresponding Author: Avinash Jayaswal, Department of Emergency

Medicine, Erasme Hospital - ULB, Brussels, Belgium.

DOI: 10.31080/ASGIS.2020.03.0123
Received: December 31, 2019

Published: February 17, 2020

(C) All rights are reserved by Avinash

Jayaswal and Mohamed El Koulali.

\section{Abstract}

Gastropericardial fistula is a life-threatening complication which results from a communication between the stomach and the pericardium. The resulting cardiac tamponnade and shock can lead to death. We present an unusual case of a 55 year-old woman who was found to have pericardial and bilateral pleural effusion on CT-scan and US imaging post-gastric bypass surgery. Endoscopy and contrast imaging showed that a fistula had developed from ischemia of the proximal eso-jejunal suture, which resulted in a para-gastric abscess communicating with pericardial and pleural spaces in the thoracic cavity. She developed ARDS and hypovolemic shock and was admitted to the ICU. Deployment of a synthetic trans-oral prosthesis endoscopically allowed tight exclusion of the fistula from the luminal cavity. A positive type Streptococcus as well as Candida albicans were respectively identified in the fluid specimen during surgical drainage. Broad spectrum antibiotics and anti-fungal agents were administered together with colchine and aspirine for pericarditis treatment. She managed to leave hospital on day 58 with a follow-up appointment 4 weeks after.

Keywords: Gastric Bypass Surgery; Pericarditis; Fistula; Endoscopic Fistula Treatment; Plastic Prosthesis; Obesity Treatment

\section{Introduction}

Gastropericardial fistula is a life threatening and abnormal communication between the stomach and the pericardial sac [1]. As bariatric surgery becomes more routine, rare complications such as enteropericardial fistula also become more likely and must be included in the differential diagnosis. Different etiologies include previous gastric or esophageal surgery, refractory gastric ulcers, trauma, tumour perforation, infection, presence of a foreign body, and caustic agent ingestion [2]. The most common presentation is a complication of prior surgery involving the stomach or the esophagus [2]. It usually occurs within a hiatal hernia and rarely occurs transdiaphragmatically [1]. Clinical findings, while inconsistent, include left shoulder tenderness due to diaphragmatic or pericardial irritation, pyrosis, epigastric pain, dyspnea and tachycardia [3-6]. While clinical presentation may vary, signs and symp- toms of septic shock are often present [2].

Depending on the type of fistula and the length of time of pericardial involvement, the amount of liquid or other substance found will differ and the diagnostic tool used will differ as well. Imaging is gold standard for workup of enteropericardial fistula guiding both emergency and surgical management [3]. Cardiac ultrasound is the standard of care to study the pericardial sac followed by computed tomography as they both have high sensitivity. A cardiac tamponnade, however unlikely, requires emergent intervention and electrocardiogram findings are then also typical [2]. Surgical intervention is the classic treatment as patients rarely survive with conservative management [3]. We report here a case of successful yet conservative management of gastropericardial fistula and pericarditis. 


\section{Clinical Case Presentation}

A 55 year-old woman was transferred to our center from a local hospital in pre-septic shock post-gastric bypass surgery. She was at post-operative day 10 and was transferred directly to ICU. Her condition degraded after being admitted for surveillance postdrainage of a para-gastric fistula and abscess post-surgery. She had undergone gastro-jejunal bypass surgery, which was a revision of the intial intervention of gastric bypass surgery by conventional laparoscopy 23 years ago. Prior to that, she underwent vertical gastroplasty as well as ring placement which worked for about 10 years before relapsing.

Post-operative outcome was complication-free and the patient was allowed oral feeding at day 2 . At day 3 , the patient started having dyspnea and oxygen saturation levels began to falter despite nasal oxygen. She also had tachycardia but no abdominal tension nor fever. Laboratory investigations showed CRP levels of $400 \mathrm{mg} /$ $\mathrm{dL}$. The abdominal drains showed a pus-like substance. A thoracoabdominal CT-scan was ordered which revealed bilateral pleural effusion and an intra-abdominal abscess and normal pericardium. Antibiotic treatment was initiated with ciprofloxacine and metronidazole due to penicilline allergy. At first, there was a mild clinical and biological improvement but CT-scan done 3 days later showed an increase in the size of the abscess. It was hypothesized that the gastric suture had failed and a fistula had formed communicating with the abscess, hence mandating the revision surgery for drainage. Oral methylene-blue test was positive confirming fistula formation. She was referred to the endoscopy team for deployment of an endoprosthesis to cure the fistula.

During the ICU transfer, her vital parameters remained stable. The patient underwent contrast oeso-gastro-duodenoscopy to confirm the presence of a fistula. Ischemia at the junction of the proximal staple line at the level of the angle of His was the origin of the fistulisation. An Ultraflex $23 / 28 \mathrm{~mm}$ was deployed to tightly exclude the fistula. The patient's condition degraded with an episode of acute respiratory distress syndrome and bilateral pleural effusion. She was put on total parenteral nutrition and recovered quite quickly to be discharged to the ward 2 days later.

Upon arrival at the ward, she had an episode of fever and laboratory testing showed increased CRP level. A thoraco-abdominal scan was repeated which showed a pericardial effusion as well as bilateral pleural effusions as well as an increase in size of the paragastric abscess. A cardiac ultrasound was ordered which confirmed circumferential pericardial effusion of $17 \mathrm{~mm}$ of thickness except at the apex, which significantly compromised mitral flow. A 10-day conservative trial with colchicine and aspirine was prescribed before a repeat ultrasound to evaluate whether an invasive treatment was necessary. The infectious disease consultant decided for an upgrade of wider spectrum antibiotics namely meropenem, vancomycin and caspofungin.

The 10-day conservative treatment was without complication. The pericardial effusion largely reduced except persistence of minimal infero-latero-basal and anterior layers. Hemodynamic parameters normalized as well as vital parameters remained within normal range. Laboratory values steadily returned to normal levels. Colchicine and aspirine were pursued with a step-down strategy. Antibiotics were maintained as well. The para-gastric abscess persisted and trials of US-guided and CT-scan guided percutaneous puncture were futile. It was thus decided to slightly pull on the abdominal drain by a few $\mathrm{cm}$ in order to optimize placement and thus successfully emptying the abscess.

\section{Discussion and Literature Review}

The first case of pneumopyopericardium caused by subphrenic abscess due to gastric ulceration was described by Hallin in 1863 [1]. Subsequently, in 1947, Harp and colleagues published the first description of a gastric communication between the stomach and the pericardial sac [1]. They described the perforation of a gastric tumor into the pericardial sac. A total of 66 cases have been described until 2016 [1]. In some cases, the patients presented with these symptoms for months and even years before consulting [1].

For patients typically diagnosed with gastro-pericardial fistula, typical investigational findings in the literature include ECG and computed tomography. We enhanced the radiographic investigation with the use of oral contrast medium, which revealed the esojejunal ischemia at the suture level as the starting point of the fistula communicating with the abscess and subsequently extending to the thoracic cavity. Cardiac ultrasound performed by the attending cardiologist was diagnostic of the extent of pericardial involvement and the decision was taken to try conservative management. Oeso-duodenal gastroscopy was carefully performed, in spite of the possible risk of pneumo-pericardial tamponnade, in order to localize the starting point of the fistula and then placing the synthetic 
ultraflex prosthesis over it, tightly enough and allow for quick advancement with closure.

A review of all published cases reveals that aggressive procedural and surgical management has reduced mortality from $69 \%$ to $11 \%$ in the last 20 years [1]. That analysis is complex as it entails Level C evidence (expert opinion, series of case studies) [1]. In the treatment options, the exact significance of interventional endoscopy is not clear, as compared to surgery, which is the radical approach. In our case, multiple surgeries had done a lot of damage already and left the patient in a multi-morbid state. Interventional endoscopy allowed the possibility to treat the fistula with the trans-oral prosthesis completely excluding it altogether along with surgical drainage of the abscess.

Furthermore, use of broad spectrum antibiotics was well managed as there was no suppurative cardiac tamponnade. The mechanism of action of secondary purulent pericarditis is usually a thoracic extension from subdiaphragmatic sites, whether it be traumatic or of surgical origin (physician-related).

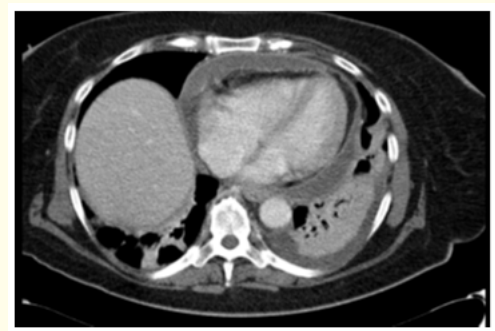

A

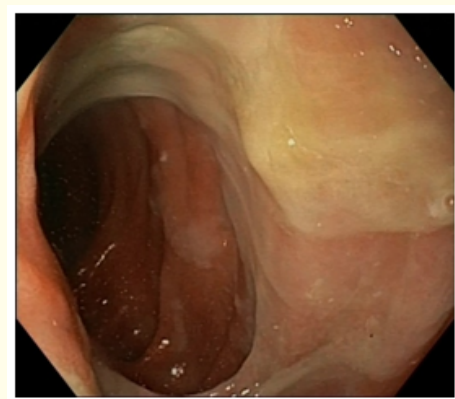

B

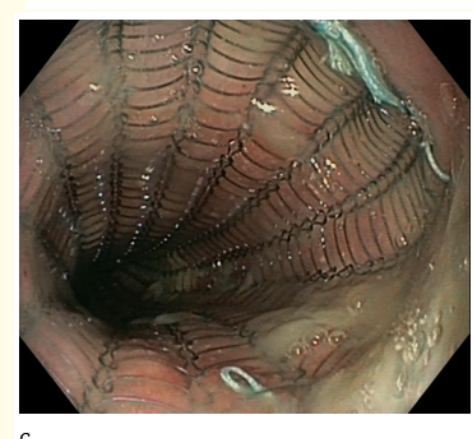

Figure 1: a: CT-scan of the heart showing the pericarial effusion, b: Endoscopy showing luminal narrowing of the esophagus and pericardioesophageal fistula, c: Endoscopic placement of tight prosthesis to exclude the fistula.

\section{Conclusion}

Gastropericardial fistula remains a rare and often fatal condition that requires prompt treatment. This study details diagnostic methods and treatment options in the management of this complication. The non-surgical approach has been less documented as the preferred method of care. We report here the full-fledged use of trans-oral Ultraflex prosthesis combined with conservative medical treatment as an option for abdominal fistula-related pericarditis.

\section{Bibliography}

1. Azzu V. "Gastropericardial fistula. Getting to the heart of the matter". BMC Gastroenterology 96 (2016): 1-5.

2. Davidson JP., et al. "Gastropericardial fistula: radiologic findings and literature review". Journal of Surgical Research 203 (2016): 174-182.

3. Chen JS., et al. "Radiologic evaluation of post-operative gastropericardial fistula”. Radiology Case Reports 9.3 (2014): 1-4.

4. Martin RA, et al. "Gastropericardial Fistula presenting 27 years after Bariatric Surgery". Clinical Practice and Cases in Emergency Medicine 1.4 (2017): 435-436.

5. Hamid UI., et al. "Closure of bronchopleural fistula using glue". Interactive Cardiovascular and Thoracic Surgery 13 (2011): 117-118.

6. Kariyanna., et al. "Esophago-pericardial Fistula Incuded Community Acquired Methicillin Resistant Staphylococcus Aureus (CA-MRSA) Cardiac Tamponade - A Rare Case Report and Literature Review". American Journal of Medical Case Reports (2018): 109-113.

\section{Assets from publication with us}

- Prompt Acknowledgement after receiving the article

- Thorough Double blinded peer review

- Rapid Publication

- Issue of Publication Certificate

- High visibility of your Published work

Website: www.actascientific.com

Submit Article: www.actascientific.com/submission.php Email us: editor@actascientific.com

Contact us: +919182824667 